Sylvie M.N. Mulliez, Thomas M. Maenhout and Carolien Bonroy*

\title{
Impact of the routine implementation of automated indirect immunofluorescence antinuclear antibody analysis: 1 year of experience
}

DOI 10.1515/cclm-2015-0900

Received September 14, 2015; accepted October 17, 2015; previously published online November 12, 2015

Keywords: antinuclear antibodies; automation; indirect immunofluorescence.

To the Editor,

Screening for antinuclear antibodies (ANA) by indirect immunofluorescence (IIF) is an important tool in the diagnosis of systemic rheumatic diseases [1, 2]. In recent years, digital systems for automated ANA-IIF analysis were introduced to overcome some of the major shortcomings of classical IIF (e.g. labor intensiveness, high inter-reader variability, risk for errors due to manual handling/reporting and the considerable expertise needed). Recently, we and others evaluated the analytical performance of these platforms in comparison with conventional IIF [3-5]. Overall, the results were promising and it was suggested that automated IIF could improve harmonization and reduce the hands-on time and risk for human error [3]. Moreover, some of the observations (e.g. the quantitative measure as an alternative for end-point titration and an objective internal quality control [IQC] measure) might offer new opportunities for the routine laboratory [6]. Here, we describe 1 year of routine experience (from June 2013 to June 2014, 4699 samples analyzed)

\footnotetext{
*Corresponding author: Carolien Bonroy, Department of Clinical Chemistry, Microbiology and Immunology, Ghent University Hospital (2P8), De Pintelaan, 185, 9000 Ghent, Belgium, Phone: +32 933236 31, Fax: +32 933249 85,

E-mail: carolien.bonroy@uzgent.be

Sylvie M.N. Mulliez and Thomas M. Maenhout: Department of Clinical Chemistry, Microbiology and Immunology, Ghent University Hospital, Ghent, Belgium
}

with an automated ANA-IIF system and evaluate whether our expectations were met.

In our center, HEp-2000 cells in combination with IgG conjugate are used (dilution 1:40) (Immunoconcepts, Sacramento, CA, USA). Slides are prepared using the PhD (Biorad, Hercules, CA, USA) or Zenit-Up (Menarini diagnostics, Florence, Italy) and analyzed automated on the Zenit G-sight (Menarini diagnostics, Florence, Italy). Zenit G-sight performs negative/positive interpretation based on the probability index (PI), the quantitative measure of the system. The PI cut-off values proposed by the manufacturer are $\mathrm{PI}<8$ (negative), $\mathrm{PI}>50$ (positive) and $8 \leq \mathrm{PI} \leq 50$ (uncertain). In our laboratory, we use adapted cut-off values: $\mathrm{PI}<8$ (negative), $\mathrm{PI}>26$ (positive) and $8 \leq \mathrm{PI} \leq 26$ (uncertain) (based on ROC analysis of both clinical as well as routine cohorts, for a description of the cohort used see Bonroy et al. 2013 [3]). Nevertheless, all samples are reviewed by expert technicians and the automated analysis results are adapted whenever necessary. As all systems are linked to the laboratory information system (LIS), data transmission is automated.

First, we evaluated to what extend the software of the system can replace the expert for negative/positive correct classification, pattern recognition and intensity scoring. See Table 1 for an overview of the PI-based positive/negative categorization of the samples in comparison with the results obtained after visual review. Based on our in-house cut-off values, expert intervention for negative/positive correct classification was needed in $49 \%$ of the samples, with most interventions needed to reclassify 'uncertains' (43\%). Expert interventions for true corrections of the system interpretation were limited (3.4\% false negatives, $2.3 \%$ false positives [based on in-house cut-off values]). When manufacturer's cut off values would have been applied, more interventions would have been needed (59\%).

Zenit G-sight is programmed to recognize five monospecific patterns [3, 4]. We compared this function with 
Table 1: Overview of the PI-based positive/negative categorization of the samples in comparison with the results obtained after visual review.

\begin{tabular}{|c|c|c|c|c|c|}
\hline \multirow{2}{*}{$\begin{array}{l}\text { Visual review of } \\
\text { digital images }^{\mathrm{a}}\end{array}$} & \multirow[t]{2}{*}{ Negative $\mathrm{PI}<8$ (\%) } & \multicolumn{2}{|c|}{ Manufacturer's cut-off values } & \multicolumn{2}{|c|}{ In-house cut-off values } \\
\hline & & Uncertain $8 \leq \mathrm{PI} \leq 50$ (\%) & Positive $\mathrm{PI}>50$ (\%) & Uncertain $8 \leq \mathrm{PI} \leq 26(\%)$ & Positive $\mathrm{PI}>26$ (\%) \\
\hline Negative & $811(84 \%)$ & $1012(39 \%)$ & $37(3 \%)$ & 939 (47\%) & $110(6 \%)$ \\
\hline Positive & $159(16 \%)$ & $1568(61 \%)$ & $1112(97 \%)$ & $1078(53 \%)$ & $1602(94 \%)$ \\
\hline
\end{tabular}

a Percentages between brackets are proportions within the PI-based subsets.

expert review of the digital images on a set of 500 consecutive positive routine samples analyzed between March and June 2014 (Supplemental Data, Table 1). Automated pattern assignment was higher in the monopattern samples $(87 \%, 316 / 362)$ compared to multiple pattern samples $(78 \%, 108 / 138)$. In $53 \%$ of the monopattern samples, automated pattern assignment needed correction (168/316). Globally, expert intervention was needed in 65\% (276/424) of the automated assigned patterns.

Based on the correlation between expert intensity scoring (negative, $1+$ to $5+$ ) on the digital images and the PI provided by the Zenit G-sight (unpublished data), we apply an automated LIS-conversion of PI into intensity scoring to limit the number of clerical handlings. Using this automated conversion no expert adjustment was needed in $65 \%(n=1844 / 2839)$ of the positive samples. Moreover, most adjustments (90\%) were minor (difference of 1 intensity).

As suggested previously, automated ANA-IIF might reduce the hands-on time [3]. In this study, we evaluated this using a random routine run of 36 samples ( 3 slides, dilution 1:40). We observed that the hands-on time for reading of the digital images was more time consuming (7 min/36 samples) than the manual ANA-IIF microscopy. In contrast automated data transmission resulted in a gain of $25 \mathrm{~min}$.

Next, we reviewed our IQC management approach. The IQC approach implemented in our center uses both IQC material (a negative and positive control [NC and $\mathrm{PC}]$ ) as well as patient data (monitoring of the median PI values per run) and is based on Westgard multirules [6].

Between June 2013 and June 2014, 146 NC and PC measurements were performed. The PI results of the NC and PC samples are plotted in Figure 1. For the NC sample there were two isolated positive violations of the control rules, one $+1_{\mathrm{S} 2}$ warning rule (April 1st) and one $+1_{\mathrm{S} 3}$ (April 8 th) rejection rule. Further investigation of the raw images used for PI calculation revealed that in both an artifact falsely influenced the PI. For the PC, there were between June 2013 and mid-October 2013 several violations of the warning and rejection rules, some of them related to technical problems with our pipetting device as previously described in more detail [6]. Based on these observations, our pipetting device was put out of order resulting in a clearly better reproducibility of the PI measurements of the PC. Only one violation was observed after October 2013, which was traced to an air bubble due to low control sample volume.

From June 2013 to June 2014, the daily median PI was 14 times outside the +2SD limit of the overall mean, three of them being major events. The first major event occurred in December 2013 and showed consecutive positive violations of the proposed control rules (four $+1_{\mathrm{S} 3}$ and one $+2_{\mathrm{S} 2}$ rejection rules). During this period no violations were observed for the PC or NC samples. Nevertheless, an increase in the \%ANA positives was seen in December 2013 (71\%) compared to September 2013 (55\%; $\left.\chi^{2} \mathrm{p}<0.0001\right)$ and October 2013 (64\%; $\left.\chi^{2} \mathrm{p}=0.034\right)$. The increase was not due to a shift in the pathology grade, because the number of samples originating from the rheumatology department was even less during the violations (19\%, 80/427) compared to stable periods (28\%, 790/2851) [6]. We further investigated whether other variables (e.g. the introduction of new reagent lot or a new vial of conjugate with the same lot, the pipetting station) might have influenced the PI measurements. Indeed, this major event was linked to the switch of the pipetting device (from Zenit Up to PhD).

After January 2014, two isolated major violations $\left(1_{\mathrm{S} 3}\right)$ on the daily median PI occurred, respectively in March and May 2014, with a parallel increase of the \%ANA positives (76\% and $64 \%$, respectively). During these violations, control measurements were normal. The violation in March could not be linked to a shift in pathology grade as the proportion of samples originating from the rheumatology department was only $21 \%$ (8/38). In contrast, in May we observed more rheumatology requests (36\%). However, this proportion was not statistically higher than the pathology grade over several periods outside events $\left(28 \% ; \chi^{2} p>0.05\right)[6]$.

To conclude, the implementation of automated ANA-IIF analysis in our routine laboratory resulted in 

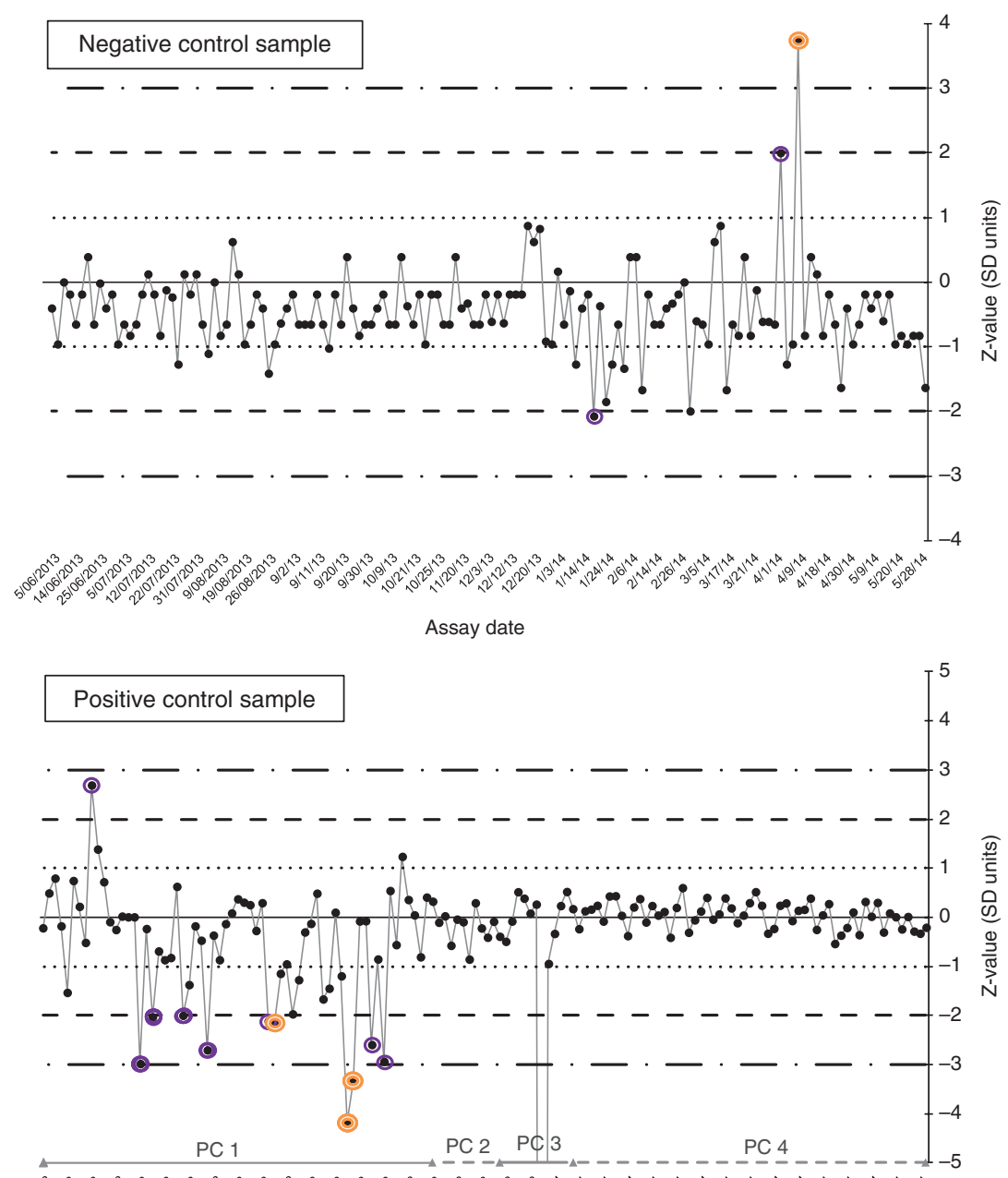

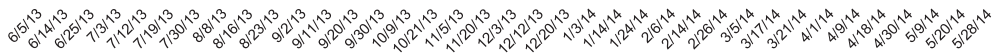

Assay date

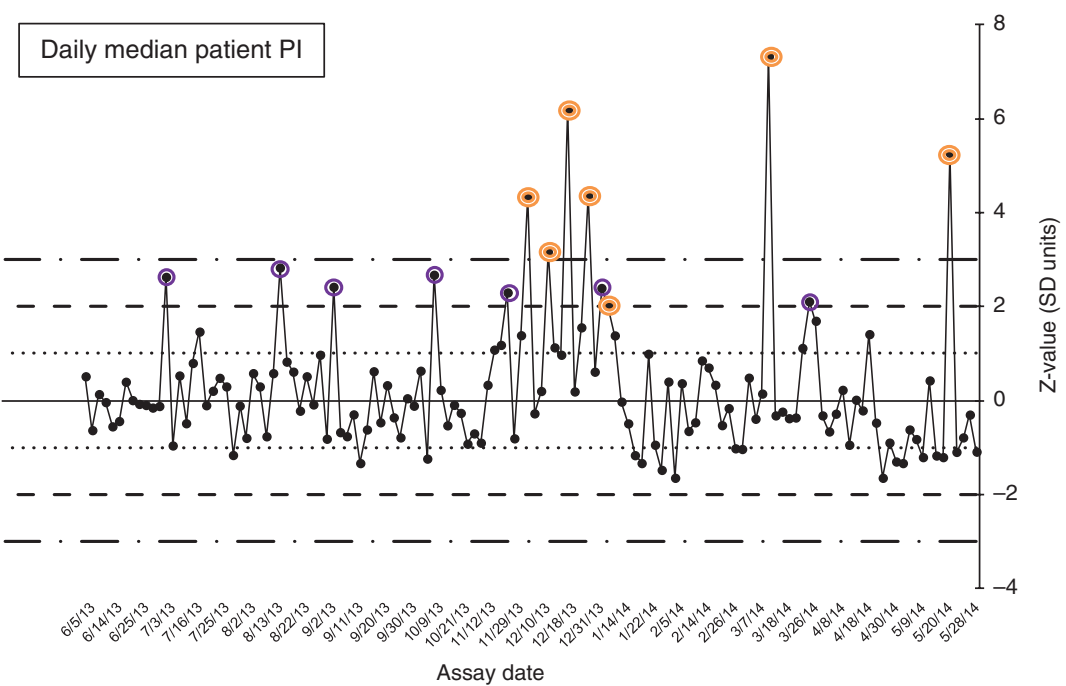

Figure 1: Levey-Jennings chart of the probability index (PI) of the negative control (NC), positive control (PC) samples, and daily median of patient PI.

Target value of PC1, PC2, PC3 and PC4 are respectively 87.6, 89.8, 89.6 and 89.4. The right $y$-axis indicates the Z-score of the PI against the target value. Single open circles indicate a $1_{\mathrm{S} 2}$ violation (warning rule); double open circles indicate a $2_{2 \mathrm{~S}}$ or $1_{3 \mathrm{~S}}$ violation (rejection rule). Horizontal lines indicate the target mean and standard deviation (SD) limits. 
important improvements in the context of expert burden and hands-on time. Expert intervention was necessary in $49 \%$ of the samples for positive/negative interpretation and in $65 \%$ of the automatically assigned patterns.

Moreover, PI conversion into intensity scoring could be automated with only limited expert correction needed. We also showed an important reduction in hands-on-time compared to the classic ANA-IIF microscopy, mainly as a result of automated data transmission. The latter also implies a reduced risk for clerical errors. Finally, our results also illustrate the practical applicability of the previously proposed quantitative IQC approach. Using this approach in a routine setting, we observed only limited number of major violations and more important, all were shown to indicate relevant changes in the total process with need for further investigations to guarantee the quality of the results.

Author contributions: All the authors have accepted responsibility for the entire content of this submitted manuscript and approved submission.

Research funding: None declared.

Employment or leadership: None declared.

Honorarium: None declared.

Competing interests: The funding organization(s) played no role in the study design; in the collection, analysis, and interpretation of data; in the writing of the report; or in the decision to submit the report for publication.

\section{References}

1. Solomon DH, Kavanaugh AH, Schur PH. Evidence-based guidelines for the use of immunologic tests: antinuclear antibody testing. Arthritis Rheum 2002;47:434-44.

2. Agmon-Levin N, Damoiseaux J, Kallenberg C, Sack U, Witte T, Herold $M$, et al. International recommendations for the assessment of autoantibodies to cellular antigens referred to as antinuclear antibodies. Ann Rheum Dis 2014;73:17-23.

3. Bonroy C, Verfaillie C, Smith V, Persijn L, De Witte E, De Keyser F, et al. Automated indirect immunofluorescence antinuclear antibody analysis is a standardized alternative for visual microscope interpretation. Clin Chem Lab Med 2013;51:1771-9.

4. Bossuyt X, Cooreman S, De Baere H, Verschueren P, Westhovens R, Blockmans D, et al. Detection of antinuclear antibodies by automated indirect immunofluorescence analysis. Clin Chim Acta 2013;415:101-6.

5. Bizzaro N, Antico A, Platzgummer S, Tonutti E, Bassetti D, Pesente $\mathrm{F}$, et al. Automated antinuclear immunofluorescence antibody screening: a comparative study of six computer-aided diagnostic systems. Autoimmun Rev 2014;13:292-8.

6. Maenhout TM, Bonroy C, Verfaillie C, Stove V, Devreese K. Automated indirect immunofluorescence microscopy enables the implementation of a quantitative internal quality control system for anti-nuclear antibody (ANA) analysis. Clin Chem Lab Med 2014;52:989-98. 